\title{
A study of an active steering bogie system for the curve negotiation on a sharp curved track
}

\author{
K. S. $\operatorname{Sim}^{1}$, H. M. Hur ${ }^{2}$, T. W. Park ${ }^{1} \&$ M. J. Kim ${ }^{1}$ \\ ${ }^{1}$ Department of Mechanical Engineering, \\ Ajou University, Republic of Korea \\ ${ }^{2}$ Korea Railroad Research Institute, Republic of Korea
}

\begin{abstract}
In this paper, analysis for reducing lateral forces is implemented by using a railway vehicle model on a sharp curved track. Also, in order to minimize some problems when a railway vehicle runs on a sharp curved track, an active-steering bogie system is investigated. An active steering bogie system of a railway vehicle is designed to alleviate contact forces and to decrease wear of wheel/rail interaction. This paper describes a modelling of an active steering and a control system for curving performance. Results of simulation, which are lateral forces and wear number of wheels, show more improvement than conventional railway vehicle. Proposed model is more effective on a sharp curved track.

Keywords: active steering bogie, sharp curve track, lateral force, curving performance.
\end{abstract}

\section{Introduction}

Urban railway vehicles operate in downtown areas. Due to increases in the number of passengers and changes in the service plans, railway vehicles are expected to operate on sharp curved track. The conventional urban trains, however, have a $1^{\text {st }}$ suspension is very stiff because of running stability so, the performance of steering of the trains is limited on curved track. Also, these trains, the lateral forces as a result of wheel/rail contact interaction are significantly increased. These phenomena causes wear of a wheel/rail, noise and vibration, etc.

In order to improve curving performance of a railway vehicle, concepts of steering mechanism are presented for a long time [1]. One commercialized steering bogie is forced steering bogie which is only composed of links and 
joints. This concept of mechanism is basic and early stage of developed system for steering. Wheel-sets can be steered by the connection element when the links between a car-body and a bogie frame are activated on the curving negotiation. The performance of this concept, however, is limited according to circumstances because of the passive element.

In order to secure more effective performance of steering on a curved track, the concepts of steering system are proposed by several researchers $[2,3]$. The active steering systems need suitable control strategies. The direct method for steering has more effective control for steering than the indirect method.

The aim of the research is to assess the ability of an active steering system to reduce lateral forces and wear and so on. Also, this paper includes results of the selected control strategies with simple control law.

\section{Model of railway vehicle}

This section discusses the full scale MBS (multi-body system) model of a railway vehicle and an active steering bogie system.

\subsection{Model of railway vehicle dynamics}

In this paper, a conventional train using a numerical model is shown in fig. 1. This model consists of single car-body and two bogies. Dynamic characteristics of a conventional train in service are applied to MBS simulation tool. The suspension is divided into a primary suspension system and a secondary system. A primary suspension consists of axle springs which are connects to a wheel-set and a bogie frame. A secondary suspension consists of air springs and dampers. These suspension elements connect a bogie frame with a car body. Also, a center-pivot connects them and provides additional degrees of freedom each other. This system is implemented as a kinematic and compliance structure. The bogie model is shown in fig. 2 .

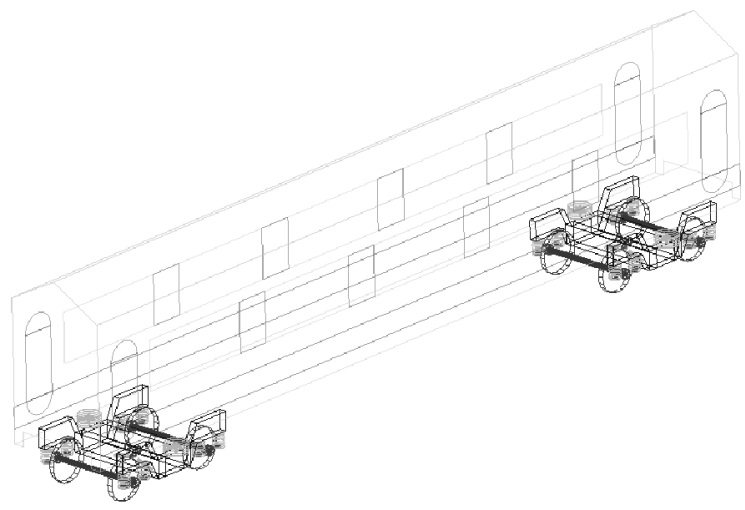

Figure 1: Full scale model of a railway vehicle. 


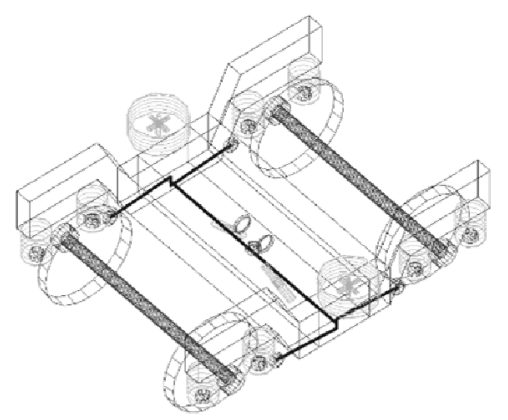

Figure 2: A bogie model of a railway vehicle.

\subsection{Concept of active steering}

The active steering methods are classified with direct method and indirect method. The direct method is that actuator forces are applied to wheel-sets. This method includes ASW (actuated solid wheel-set), AIRW (actuated independently wheel-sets), DIRW (driven independently rotating wheel-set) and DSW (directly steered wheel-set) and so on. The indirect method is that actuator forces aren't applied to wheel-sets but applied to a bogie frame and so on. This method includes secondary yaw control.

Direct methods for steering control are lateral position control and yaw-moment control of wheel-set as shown in fig. 3 and fig. 4 respectively [4]. In this paper, the yaw moment control of wheel-set is proposed.

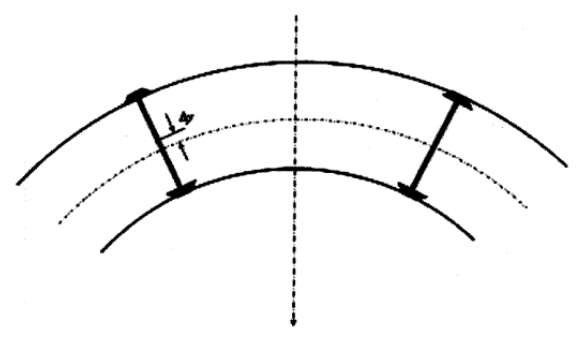

Figure 3: Lateral direction of wheel-set in a curve.

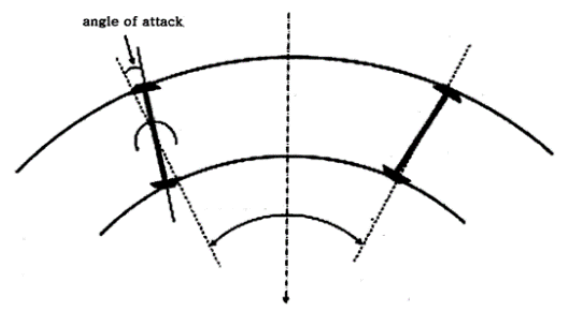

Figure 4: Radial position of wheel-set in a curve. 


\section{Dynamic analysis on curved track}

\subsection{Condition of analysis}

In order to make a comparison of a conventional vehicle and an active steering vehicle, a sharp curved track is modelled as shown in fig. 5. The radius of curved track is $300 \mathrm{~m}$, the length of transition curve is $200 \mathrm{~m}$ and the cant is $152 \mathrm{~mm}$.

The running speed of a simulation model is constant speed of $70 \mathrm{~km} / \mathrm{h}$ between a straight and a curved track.

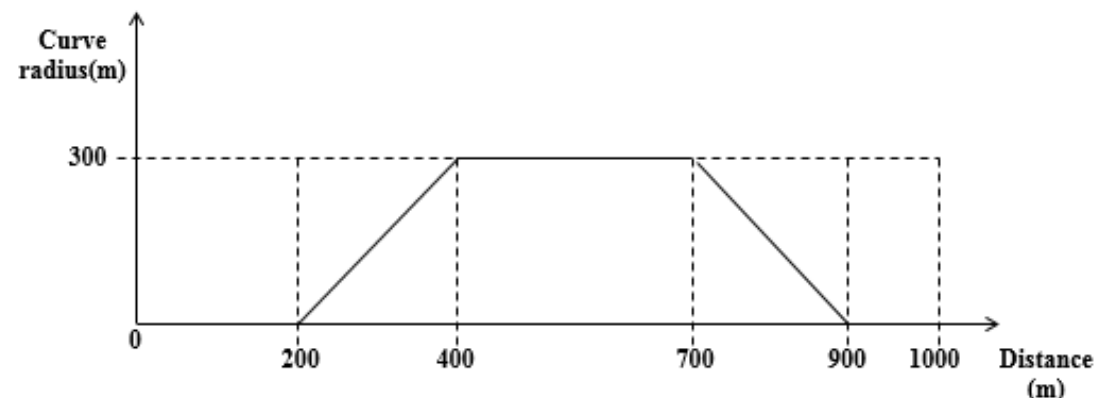

Figure 5: Definition of small curved track.

\subsection{Results of analysis}

The dynamic analysis is performed in VI-Rail and MATLAB/Simulink to investigate the curving negotiation of active steering on curved track. A vehicle model which is plant model is modelled using VI-Rail and control model is modelled using MATLAB/Simulink. In this paper, an active steering model is applied to PID control. The control strategy is directly modelled in MATLAB/Simulink. Co-simulation is performed by this software.

The results of simulation between a passive vehicle and an active steering vehicle are shown as figs. 6-11. The lateral forces are compared in fig. 6 and fig. 7. The wear numbers of wheel/rail are compared in fig. 8 and fig. 9. The derailment quotients are shown in fig. 10 and fig. 11 . The outer side of the curve is the left side of vehicle's running direction and the inner side of the curve is the right side of vehicle's running direction. Thus, the front left wheel of front bogie yielded the highest deviation of the lateral force, derailment quotient and wear. In the front wheels, the lateral forces of an active steering vehicle is reduced more than 30 percent as compared with the lateral force of passive vehicle. Also, the wear and derailment index of railway vehicle are improved by active steering system. 

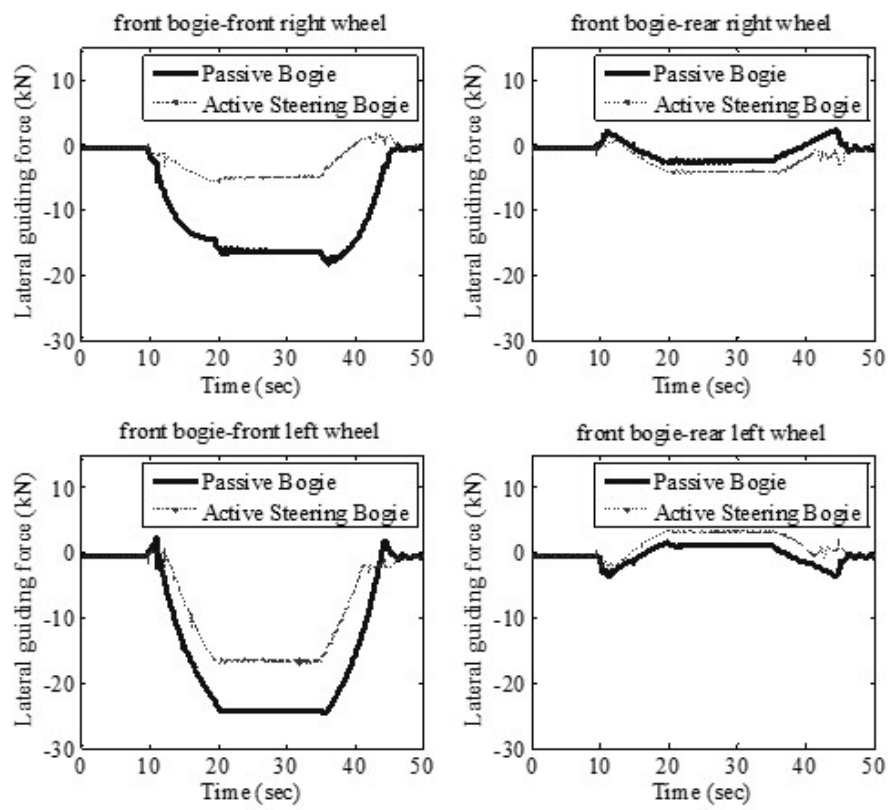

Figure 6: Lateral forces of a front bogie.
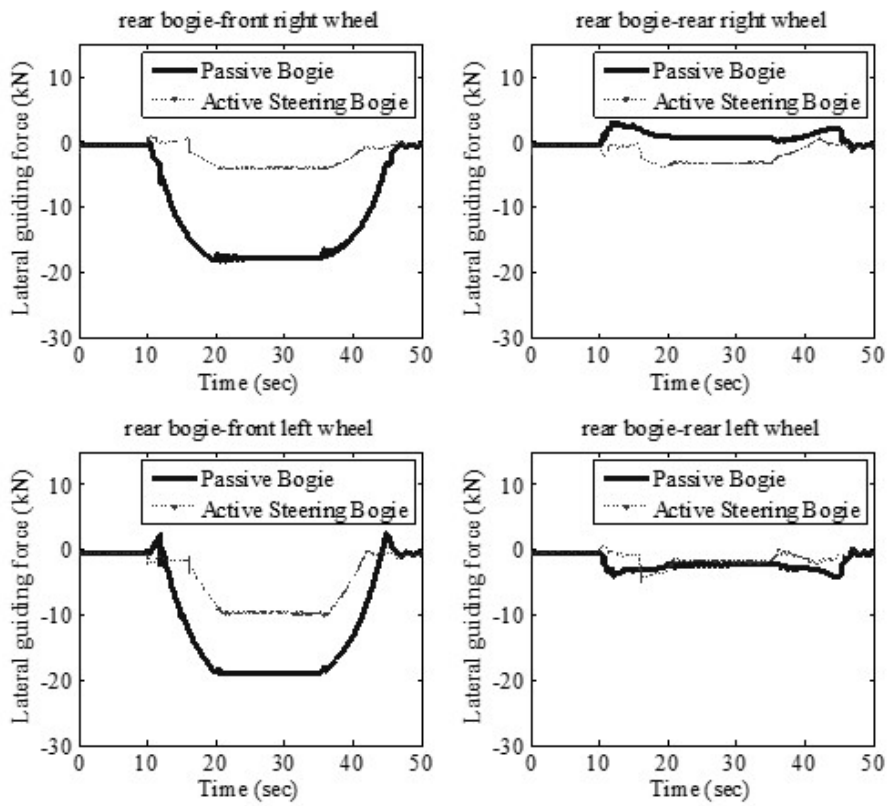

Figure 7: Lateral forces of a rear bogie. 

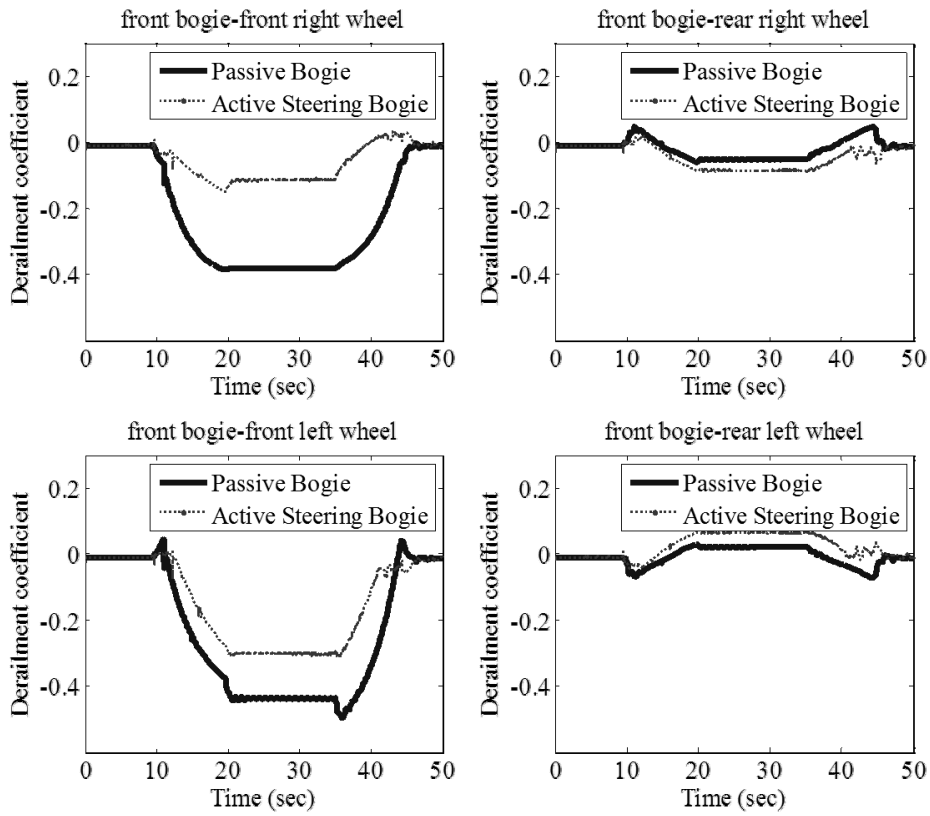

Figure 8: Derailment quotient of a front bogie.
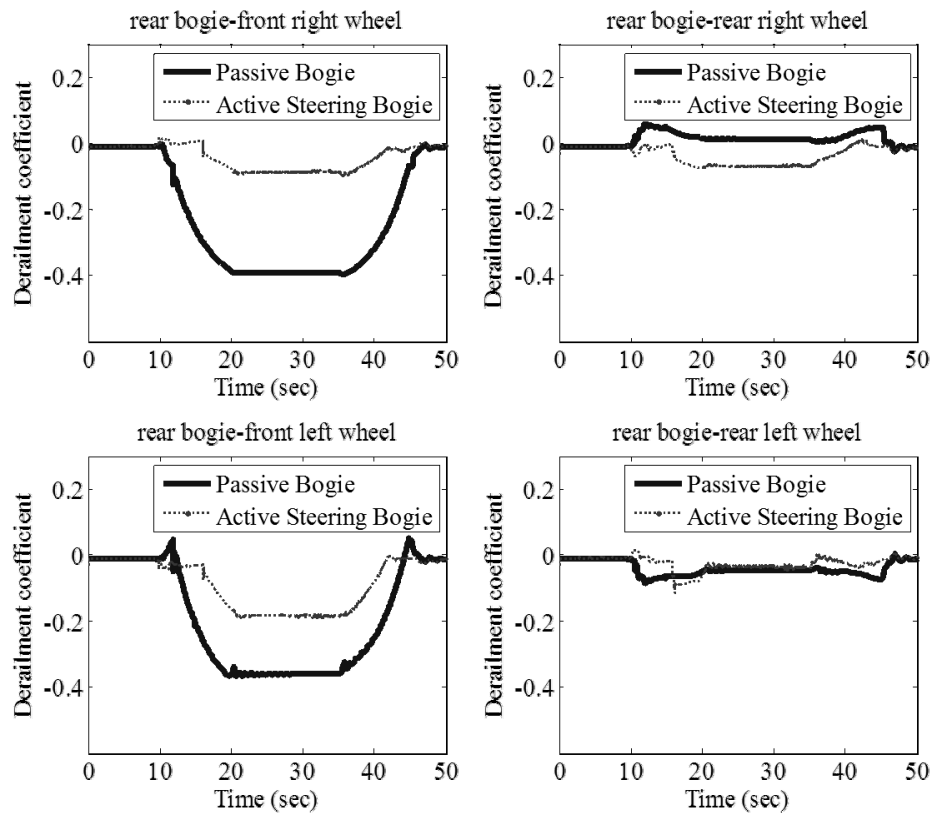

Figure 9: Derailment quotient of a rear bogie. 

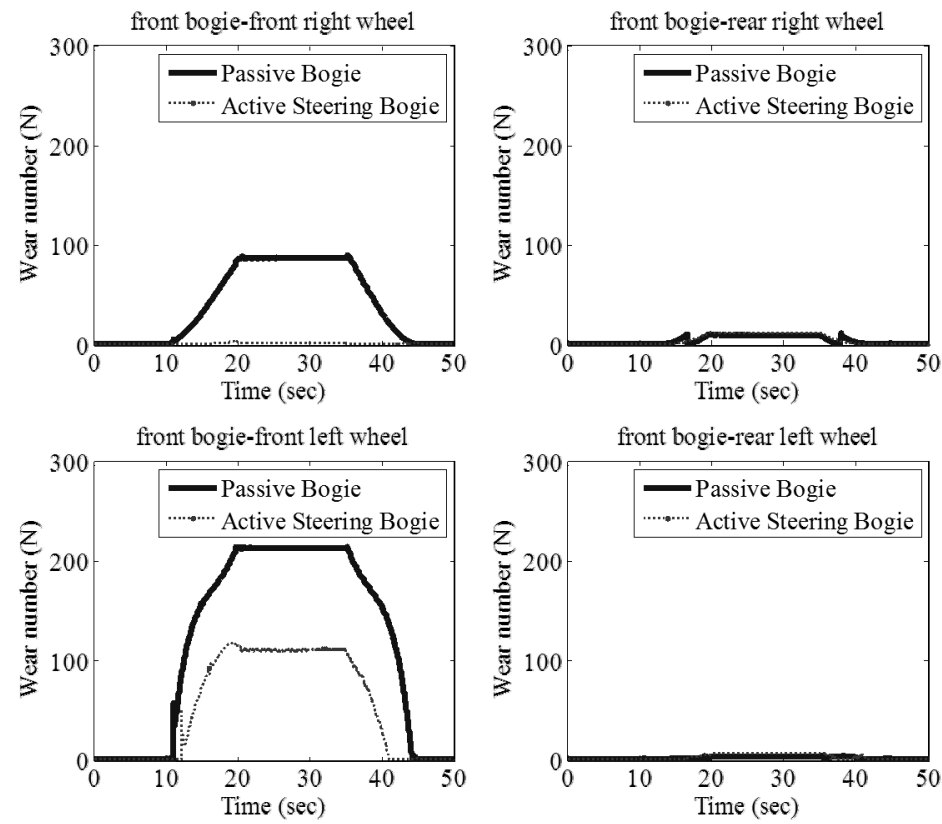

Figure 10: Wear number of a front bogie.
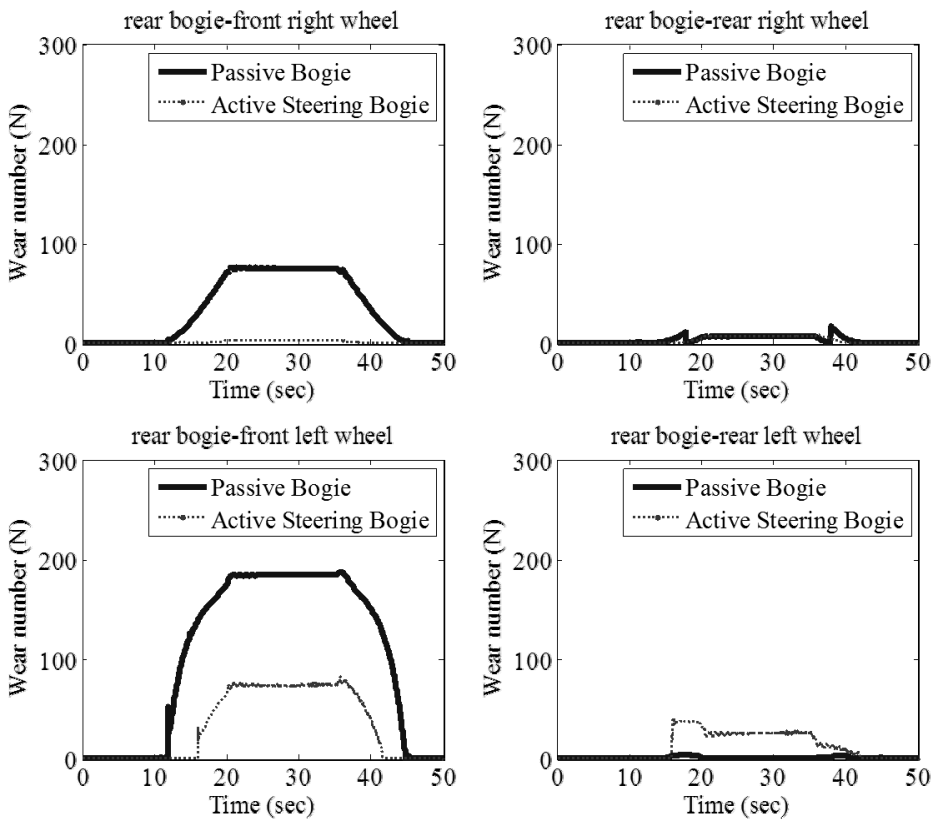

Figure 11: Wear number of a rear bogie. 


\section{Conclusion}

In this study, an active steering bogie system that accounts for the effect of the curving negotiation is developed and integrated into general multi-body system. Furthermore, the simple control law which is PID control is integrated into multi-body code using co-simulation techniques, it is proposed for an active steering performance. The active steering bogie enables the value of lateral forces of front wheel-sets to be reduced even though the bogie runs on small curve. Also, derailment quotient and wear index are decreased on curved track. So, in order to reduce lateral force and so on, an active steering vehicle is more effective than passive vehicle. Also, active steering bogie is expected to cost down of maintenance of wheel/rail and secured with safety getting an increase in speed. In the future, simulation model of active steering system will be evaluated on actual track data. In addition, an active steering model will be developed.

\section{Acknowledgement}

This research was supported by a grant (13RTRP-B072165-01) from the Railroad Technology Research Program funded by the Ministry of Land, Infrastructure and Transport of the Korean government.

\section{References}

[1] Smith. R. E., Performance testing of the Resco steered frame freight car truck at the AAR test and comparisons with standard and premium three-piece trucks. Resco engineering project report.

[2] Mei. T. X. \& R. M. Goodall, Wheelset control strategies for a two axle railway vehicle. $J$. Vehicle system dynamics, 33, pp. 653-664, 2000.

[3] Pearson. J. T., R. M. Goodall, Mei. T. X. \& Himmelstein. G., Active stability control strategies for a high speed bogie. Control Engineering Practice, Elsevier, 12, pp. 1381-1391, 2004.

[4] Hur, H. M., You, W. H., Park, J. H., Kim. M. S. \& Kim N. P., Active steering control strategy of railway rolling-stock using the estimated curvature of curve section. Proc. of spring of Korea Society of Mechanical Engineers, pp. 326-327, 2010. 